\title{
UAV Path Planning Based on Split Method
}

\section{Zhi-Ming GU ${ }^{1,2, a}$, Wen-Ming GAO ${ }^{1,2, b}$, Xu-Sheng GAN ${ }^{1,2, c}$} ${ }^{1}$ Air Traffic Control and Navigation College, Air Force Engineering University, Xi'an, Shaanxi, 710051,
China

${ }^{2}$ National Key Laboratory of Air Traffic Collision Prevention, Xi'an, Shaanxi, 710051, China

aemail: 41829292@qq.com, bemail: 472728964@qq.com, cemail: ganxusheng123@163.com

Keywords: UAV; Ant Colony Algorithm; Path Planning; Split Method

\begin{abstract}
Path planning technology is the precondition of unmanned aerial vehicle (UAV) airspace safety. For in the running features of the UAV in 3-D space environment, putting forward using fight level (FL) guiding factor to improve the basic ant colony optimization(ACO), which improved the quality and the operation efficiency of path planning; In view of the ACO which easily fell into local optimum in huge space, putting forward using split method to make the improved ACO have a better effect in huge path planning problem; Through simulation experiments, the basic algorithm and improved algorithm and split method were analyzed, the results show that the improved algorithm has a good 3-D path planning ability.
\end{abstract}

\section{Introduction}

Problem of the path planning in a threat environment is well known but stills to be in the focus of research[1]. Ant colony algorithm(ACO) was first proposed by Dorigo.M In 1991, and successfully applied to the TSP problem[2], scheduling problem, packing problem. The path planning problem is essentially a kind of combinatorial optimization problem, and the ant colony algorithm has achieved some results in this field.

In this paper, according to the characteristics of UAV airspace operation, FL and target points are used as a heuristic factor to improve ACO. Because the ACO is easy to fall into local optimum[3], space partition method is proposed to improve the quality of path planning.

\section{Improved ant colony algorithm}

In the traditional ACO, the distance between the node and the target point is generally used as the heuristic information, as shown in the formula[4]:

$\eta_{n}=1 / d_{n}$

Where $\eta_{n}$ is ant visibility, $d_{n}$ is the distance between the node and the target point.

In this paper, the definition of the heuristic information in the 3D space is improved according to the operating characteristics of UAV. In the UAV real airspace operation state, the UAV will fly in the preset FL, so UAV should be guided into the preset FL, and then move to the target point, as shown in the formula:

$$
\begin{aligned}
& \eta_{n}=1 /\left(d_{n}+\Delta l\right) \\
& \Delta l=\left|l_{g}-l_{j}\right|
\end{aligned}
$$

Where $l_{g}$ is the height of the target point, $l_{j}$ is the height of the node.

An ant $k$ at node ${ }^{i}$ selects the next node $j$ to visit with probability[5]:

$$
P_{i j}^{k}(t)= \begin{cases}\frac{\left[\tau_{j}(t)\right]^{\alpha} \times \eta_{j}^{\beta}}{\sum_{s \in a l l o w e d}\left[\tau_{s}(t)\right]^{\alpha} \times \eta_{s}^{\beta}} & \text { if } j \in \text { allowed }_{k} \\ 0 & \text { else }\end{cases}
$$


Where $\tau_{j}(t)$ is the level of node $j$ pheromone, $\alpha$ and $\beta$ are two parameters that determine the relative importance of the pheromone with respect to the heuristic information. allowed ${ }_{k}$ is the set of neighbor nodes that have not yet been visited by the ant $k$.

Introduce the model of MAX-MIN Ant System (MMAS) to limit the concentration of pheromone in the path. As shown in the formula:

$$
\tau_{j}(t)= \begin{cases}\tau_{\min }, & \tau_{j}(t)<\tau_{\min } \\ \tau_{\max }, & \tau_{j}(t)>\tau_{\max } \\ \tau_{j}(t), & \tau_{\min } \leq \tau_{j}(t) \leq \tau_{\max }\end{cases}
$$

The pheromone update rule is:

$$
\begin{aligned}
& \tau_{j}(t+1)=(1-\rho) \times \tau_{j}(t)+\Delta \tau_{j}^{*}+\sum_{k=2}^{\gamma} \Delta \tau_{j}^{k} \\
& \Delta \tau_{j}{ }^{k}= \begin{cases}\frac{F}{L_{k}} & \text { if } \quad j \in p^{k} \\
0 & \text { else }\end{cases} \\
& \Delta \tau_{j}^{*}=\left\{\begin{array}{lll}
\frac{F^{*}}{L^{*}}, & \text { if } & j \in p^{*} \\
0, & \text { else }
\end{array}\right.
\end{aligned}
$$

Where $\rho$ is the pheromone evaporation parameter that controls the decay of pheromone. $F^{*}$ and $F$ is constant. $L^{*}$ is the path length of the optimal ant.

After the evaluation of the adaptability of each path, the node record and the adaptive value of the optimal path are recorded. At the end of a cycle, empty the tabu list of all ants, in accordance with the new pheromone distribution to explore the path and the pheromone update, record the best path of this cycle, and compare with the history of the best path, record the new optimal path information.

\section{Path planning algorithm based on split method}

In order to simplify the airspace operation environment, reduce the search space, the planning speed, cross grid three-dimensional space is discretized using the intersection as key nodes[6]. Give the grid number, from left to right, from back to front, from the bottom to the top.

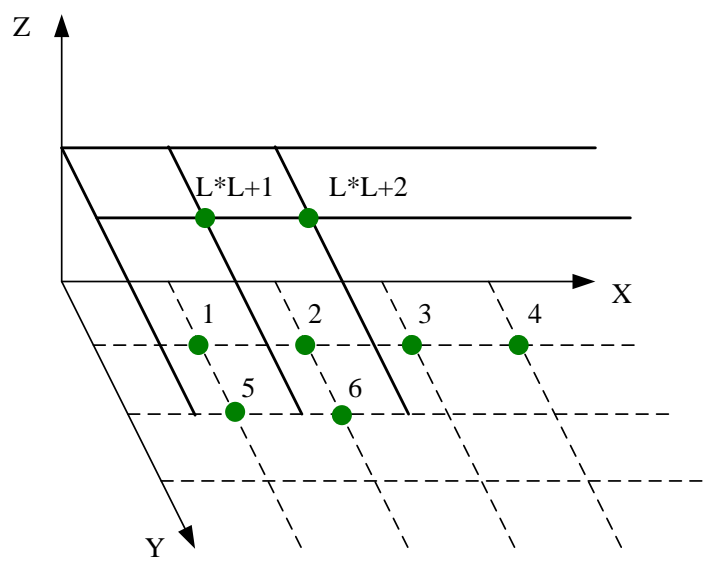

Fig.1. Spatial grid diagram

Each node number in a 3D grid map corresponds to a node's space coordinates, when the coordinates of a node is required to use the coordinates of a node, then coordinate converted between a three-dimensional coordinate information and node number.

In the face of complex spatial structure and large scale, the optimization performance of the algorithm determines the quality of the final solution, In the problem of path planning for UAV, this phenomenon is particularly evident. When the spatial scale becomes larger, the path of the redundant 
degree will be significantly increased, the smoothness of the path is reduced, and the planning time is prolonged, thus forming the non-flying path.

In this paper, an improved ant colony algorithm based on spatial segmentation is proposed, which can reduce the difficulty of the path planning by the way of dividing the space of planning and reduce the difficulty of path planning. Algorithm first generates a rough path from the starting point to the end point, which will be redundant.

The second step is to selecte the intermediate node of rough path as the splitting point,which is a starting point for the first half of the path of the endpoint.Finally, connecting the two path segments as the final path.

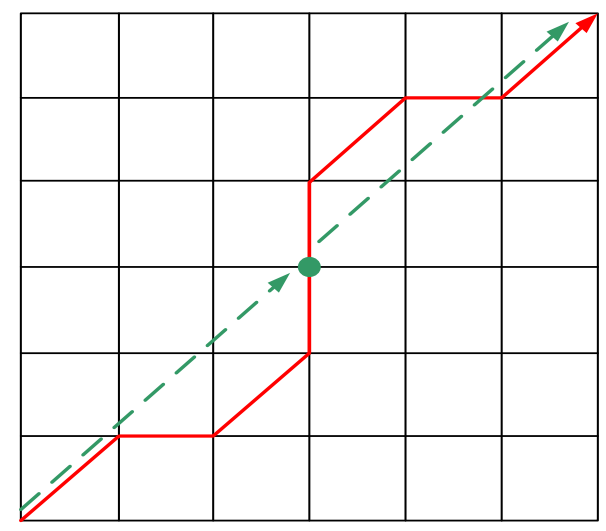

Fig.2. Sketch map of space partition method

\section{Computational results}

In this section, we present computational results of our proposed algorithm, which was code in the MATLAB2008.

Table1 Main parameters setting

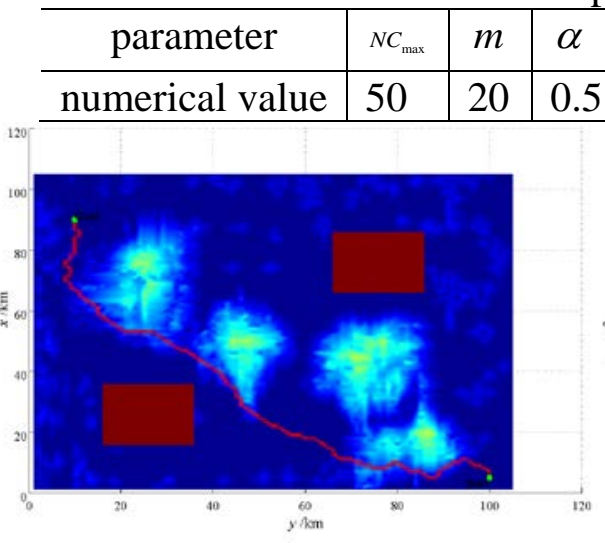

(a) $90^{\circ}$ view

\begin{tabular}{l|l|l|l|l|l|l}
\hline & $\tau_{\max }$ & $\tau_{\min }$ & $\rho$ & $F$ & $F^{*}$ \\
\hline & 160 & 20 & 0.5 & 0.8 & 100 & 50 \\
\hline
\end{tabular}

Fig.3. Path planning based on basic ACO

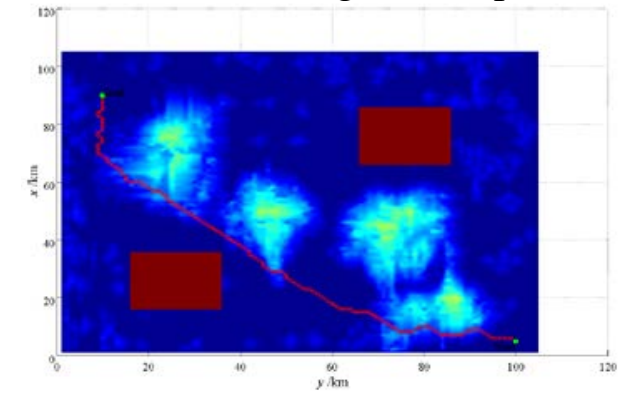

(a) $90^{\circ}$ view

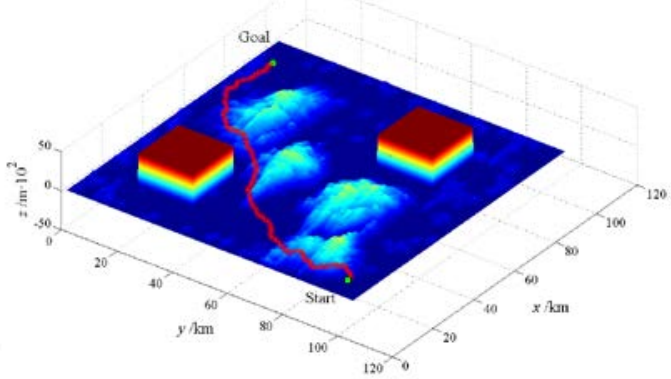

(b) $45^{\circ}$ view

Fig.4. Path planning based on improved ACO 


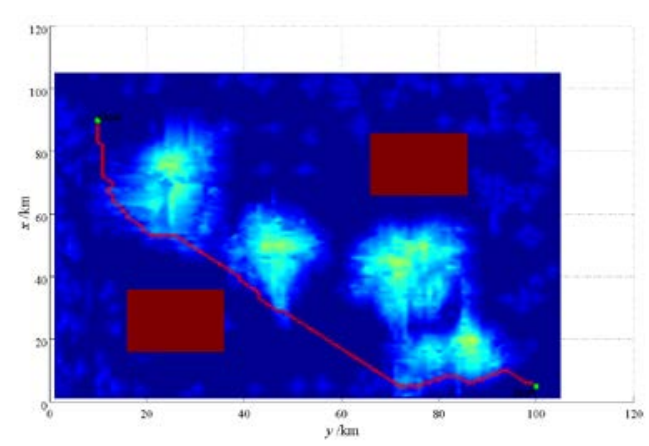

(a) $90^{\circ}$ view

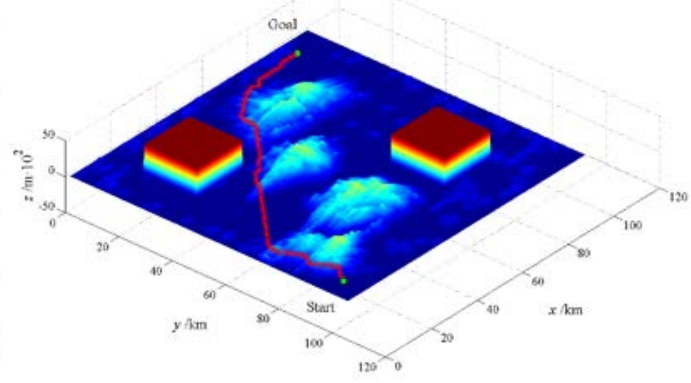

(b) $45^{\circ}$ view

Fig.5. Path planning based on spatial partitioning

Path length comparison of three algorithms: 。

Table2 comparison of algorithm

\begin{tabular}{cc}
\hline Algorithm type & Path length $(\mathrm{km})$ \\
\hline Basic ACO & 159.0687 \\
Improved ACO & 154.2478 \\
ACO based on spatial partitioning & 146.1665 \\
\hline
\end{tabular}

In the path planning, the improved ant colony algorithm based on space partition is more time than the basic algorithm, because this improved algorithm use the planning time to exchange for path planning quality, the required average is $12.7945 \mathrm{~s}$, but it has been able to meet the needs of the static path planning for real-time[7], which have been greatly improved relative to the previous research results.

\section{Conclusion}

The static path planning of UAV is mainly used to avoid some of the static obstacles or the area of the flight. In this paper, according to the characteristics of UAV airspace operation, the basic ant colony algorithm is improved by using the guiding factor level to improve the quality and efficiency of path planning. In the large scale and high complexity of the airspace operation environment, Simulation experiments show that the improved algorithm can effectively improve the path of the simplification, avoid all kinds of threat factors, to ensure the safe operation of the UAV airspace.

\section{Acknowledgement}

In this paper, the research was sponsored by the Provincial Natural Science Foundation of Shaanxi Province (Project No. 2015JM7364).

\section{References}

[1] DORIGO M. Special section on ant colony optimization [J]. IEEE Trans on Evolutionary Computation, 2002,6( 4) : 317-319.

[2] J.J Song, An improved ant colony algorithm and its application in the shortest path [D]. Taiyuan: North Central University,2013.

[3] Z.H Wang,W.G Zhang,W.G Li,UAV path planning using improved multi-objective ant colony system [J]. Application Research of Computers,2009,26(6),2104-2106.

[4] X.Z Wang,Dynamic Path Planning and Simulation for Unmanned Aerial Vehicle[D]. Nanjing: Nanjing University of Aeronautics and Astronautics,2013.

[5] F.H Jin,B.R Hong,Q.J Gao,Path Planning For Free-Flying Space Robot Using Ant Algorithm[J]. Robot. 2003,24( 6) : 526-529. 
[6] C. Mou,J. Xiao.C.S Jiang,Three dimensional path planning of UAV with improved ant algorithm[J].Journal of Jilin University,2008,38(4),991-995.

[7] Department of Defense. Unmanned System Integrated Roadmap FY2013 2038[R]. Washington DC: Department of Defense. 2013. 